\title{
Defects and Polytypism in SiC: The Role of Diffuse X-Ray Scattering
}

\author{
A. Boulle ${ }^{a}$, D. Dompoint ${ }^{a}$, I. Galben-Sandulache ${ }^{b}$, D. Chaussende ${ }^{b}$ \\ ${ }^{a}$ Science des Procédés Céramiques et de Traitements de surface (SPCTS - CNRS UMR 6638), ENSCI \\ 47 avenue Albert Thomas 87065 Limoges Cedex, France \\ ${ }^{b}$ Laboratoires des Matériaux et du Génie Physique (LMGP - CNRS UMR 5628), Grenoble INP, Minatec \\ 3 parvis Louis Néel, BP 257, 38016 Grenoble Cedex 01, France
}

\begin{abstract}
Stacking faults (SFs) and the 3C-6H polytypic transition in thick (001)-oriented 3C-SiC crystals are studied by means of diffuse X-ray scattering. The presence of SFs lying in the $\{111\}$ planes gives rise to streaked reciprocal lattice points with the streaks being parallel to the $<111>$ directions. In the case of low SF densities the defects are uncorrelated and the simulation of the diffuse intensity distribution allows to derive the SF density. In partially transformed crystals, the SFs are spatially correlated which gives rise to an intense and asymmetric diffuse scattering distribution. Its simulation allows to determine both the transformation mechanism and the transformation level.
\end{abstract}

Keywords: SiC, Defects, Diffuse X-ray Scattering

PACS: $61.72 . \mathrm{Dd}$, 61.72.Nn

\section{INTRODUCTION}

Silicon carbide is the archetype of polytypic materials with more than 200 forms referenced ${ }^{1}$. Among them, the cubic (3C-SiC) phase exhibits the most appealing properties for high-power, high-frequency and harsh environments operating devices. Despite this interest, the growth of high-quality $3 \mathrm{C}$-SiC single-crystals is still hindered by its instability at the temperatures usually required to grow $\mathrm{SiC}\left(>1900^{\circ} \mathrm{C}\right)$. This results in a strong propensity to transform into the $6 \mathrm{H}$ polytype ${ }^{2,3}$ through the formation and the extension of a large number of stacking faults (SFs) in the $\{111\}$ planes.

In the past, $\mathrm{SFs}$ and polytypism in $\mathrm{SiC}$ have been characterized by different techniques such as $\mathrm{X}$-ray rotation photography ${ }^{4,5}, \mathrm{X}$-ray diffraction (XRD) and X-ray topography (XRT) ${ }^{6}$, transmission electron microscopy (TEM) ${ }^{3,7}$ or Raman scattering ${ }^{8,9}$. Whereas an estimation of a SF density could sometimes be obtained by TEM or XRT, none of these techniques could provide any global and statistically relevant information regarding the spatial properties of the $\mathrm{SF}$ distribution (i.e. is the SF distribution strictly random or are there spatial correlations ?) neither concerning the degree of transformation of the $3 \mathrm{C}$ crystals. These informations can, in principle, be accessed by diffuse X-ray scattering (DXS). However, as in the case of XRD (and other X-ray diffraction/scattering-based methods), the interpretation of DXS data is hampered by the 'phase problem', i.e. the quantity measured experimentally is the scattered intensity (square modulus of the scattered amplitude). The direct inversion of DXS data therefore yields the autocorrelation function of the electron density within the crystal $^{10}$, and not the electron density itself which is the quantity we are actually looking for. This problem can be circumvented by fitting the experimental data with a model including the spatial properties of the electron density within the crystal. This is the topic of the present article in the case of the study of stacking faults and polytypism in 3C-SiC. We first provide details concerning DXS data acquisition. We then show that the quantitative analysis of DXS data is able to provide the SF density in the case of relatively low SF densities. In the case of high SF densities (as encountered in partially transformed 3C-SiC 
crystals), this technique allows to provide the transformation level as well as the spatial properties of the SF distribution, i.e. the transformation mechanism.

\section{EXPERIMENTAL DETAILS}

In the present study we used $10 \times 10 \mathrm{~mm}^{2}, 250 \mu \mathrm{m}$ - thick, commercially available (001)-oriented 3C-SiC single crystals (HAST Corporation) grown by chemical vapor deposition on "undulant" (001) Si wafers ${ }^{11}$. Two crystals have been investigated : an untransformed $3 \mathrm{C}$-SiC crystal and a partially transformed crystals $(1.6 \%$ transformation level). This latter crystals has then been annealed at $1700^{\circ} \mathrm{C}$ during 5 hours so as to further promote the $3 \mathrm{C}-6 \mathrm{H}$ transition.

Wide-range reciprocal space maps (RSMs) have been recorded on a home-made laboratory equipment based on a rotating $\mathrm{Cu}$ anode coupled with a four reflections $\mathrm{Ge}(220)$ monochromator and equipped with a curved position sensitive detector (PSD) with a $120^{\circ}$ angular aperture. A five-movement sample holder allows precise sample

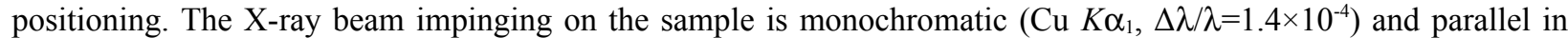
the detector plane $\left(\Delta \theta=12\right.$ arcsec) with dimensions $10 \times 0.1 \mathrm{~mm}^{2}$ so that a large volume of the sample is analyzed which provides statistically significant averaged values. A detailed description of the set-up has been given elsewhere $^{12}$. A RSM represents the scattered intensity in a particular $\left(Q_{x}, Q_{z}\right)$ plane, where $Q_{x}$ and $Q_{z}$ are the components of the scattering vector $\mathbf{Q}(Q=4 \pi \sin \theta / \lambda)$ in the film plane and perpendicular to it, respectively. In the following experiments, $Q_{x}$ and $Q_{z}$ have been set parallel to the [1-10] and the [001] directions of SiC.

The reciprocal lattice of $3 \mathrm{C}-\mathrm{SiC}$ together with the incident wave vector $\mathbf{K}_{0}$ and the associated Ewald's sphere are represented in figure 1 (the Ewald's sphere is reduced to a $120^{\circ}$-wide arc so as to match the actual angular aperture of the PSD). When the incidence angle $\omega$ is varied between $\omega_{0}$ and $\omega_{0}+\Delta \omega$ the Ewald's sphere spans the range indicated by the gray area, so that in one single $\omega$-scan we record a very wide portion of the reciprocal space, including the (-113) and (002) reflections of 3C-SiC.

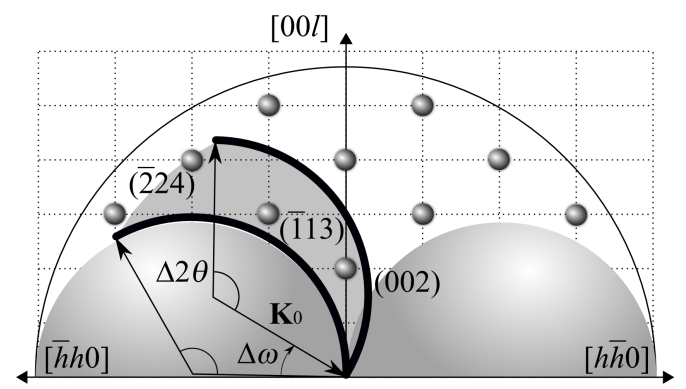

FIGURE 1. Schematic representation of the reciprocal lattice of 3C-SiC. The small gray spheres correspond to the accessible reflections of $\mathrm{SiC}$; only the measured reflections are indexed. $\mathbf{K}_{0}$ is the wave vector of the incident beam. The portion of the Ewald's sphere associated to the detector is shown by the thick $120^{\circ}$-wide arcs. The sample is rotated by $\Delta \omega$ during the experiment and the detector opening is $\Delta 2 \theta$. The two gray half-spheres correspond to regions that are not accessible in the reflection geometry, whereas the larger half-sphere corresponds to region that can be accessed with the present wavelength.

\section{RESULTS AND DISCUSSION}

\section{Low SFs density}

A RSM recorded from an untransformed sample is given in figure 2(a-b). Several features can be observed on this map. The very weak streak parallel to the normal to the surface (i.e. parallel to [001]) is due to the truncation of the crystal lattice at the surface, the so-called crystal truncation $\operatorname{rod}^{13}$. The intense streak labeled "PSD" lying along the Ewald sphere is due to the transmittance function of the $\mathrm{PSD}^{12}$. The two remaining inclined streaks make an angle $\psi \approx 55^{\circ}$ with the [001] direction. Since one-dimensional features in reciprocal space correspond to twodimensional features in real space and considering that the angle between the (001) and (111) is $\psi_{(001),(111)}=54.74^{\circ}$, these streaks can undoubtedly be attributed to planar defects lying in the $\{111\}$ planes ${ }^{14}$. Moreover, since the streaks 
have a rather weak intensity (as compared to those encountered in the next section for instance) and are symmetrical with respect to the center of the reflection it can be concluded that the SF density is low and that the SF are spatially uncorrelated, i.e. the position of a given SF has no influence on the position of the neighboring SFs.
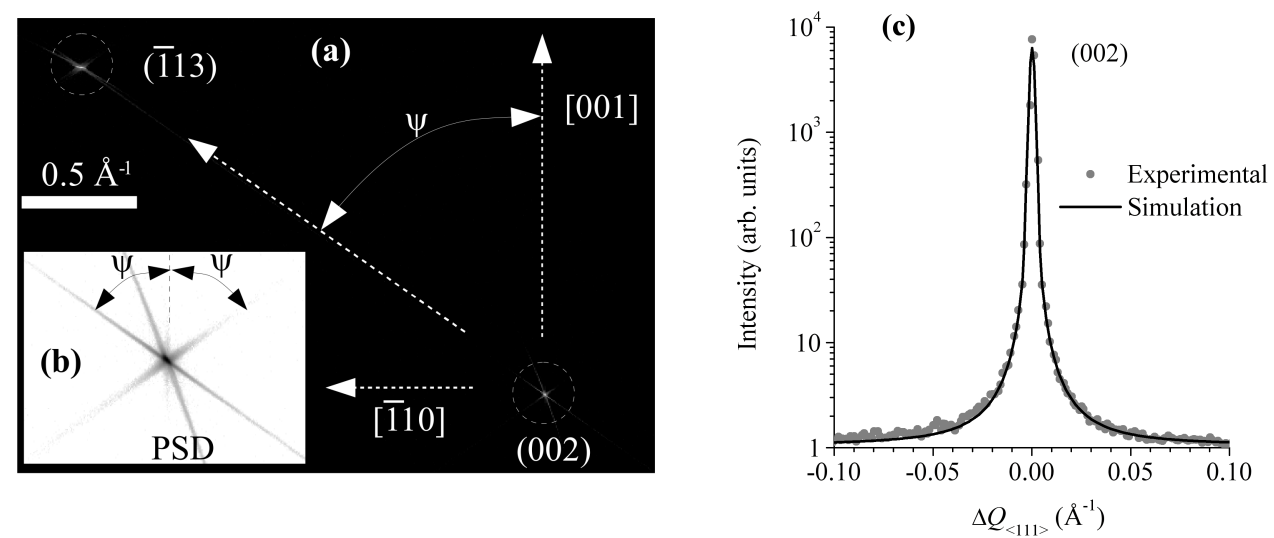

FIGURE 2. (a) wide-range RSM of an untransformed 3C-SiC crystal. The (002) and (-113) reflections are indicated. The angle $\psi$ corresponds to the angle between the [001] and [-111] direction. (b) 12x magnification of the (002) reflection evidencing the various streaks. (c) Simulation of the intensity distribution along the the diffuse streak.

Quantitative information concerning the SF density can be obtained the simulation of the intensity distribution along the diffuse streaks. This intensity distribution can be written :

$$
\left.I \mid Q_{\langle 111\rangle}\right)=\sum_{m=-\infty}^{\infty} \tilde{R}(m) V(m) G(m) \exp \left(i Q_{\langle 111\rangle} \cdot m \cdot d_{111}\right)
$$

where $d_{111}$ is the planar spacing of the (111) planes, $Q_{<111>}$ is the component of the scattering vector $\mathbf{Q}$ along a particular $<111>$ direction, $\tilde{R}$ is the Fourier transform of the resolution function of the diffractometer ${ }^{12}, V$ is the correlation volume (describing the effect of the mosaic block size and shape) ${ }^{15}$ and $G$ is the pair correlation function describing the effect of faulting. In the case of uncorrelated disorder the expression of $G$ has been given in Ref. 14, and the result of the simulation is shown in figure 2(c). It can be observed that the model nicely fits the data of four orders of magnitude and the corresponding SF density is $\rho=6.4 \times 10^{3} \mathrm{~cm}^{-1}$. It is noteworthy that a similar value $\left(6 \times 10^{3} \mathrm{~cm}^{-1}\right)$ has been obtained independently by TEM on similar samples ${ }^{7}$.

\section{High SFs density}

We now investigate the case of partially transformed 3C-SiC crystals. The corresponding RSM is given in figure 3(a-b). The most striking difference, as compared to the previous case, is that the intensity of the SF-induced streak is strongly enhanced (the (002) and (-113) reflections are now connected by a continuous intensity streak), indicating a significant increase of the SF density. Moreover, a careful examination of the intensity distribution within the streak reveals that it is no more symmetric, which is in turn indicative of spatial correlations in the SF distribution. This feature is easily understood considering the fact that the $3 \mathrm{C}-6 \mathrm{H}$ transition (which implies a restacking from $\mathrm{ABCABC}$ to $\mathrm{ABCACB}$ ) necessarily implies spatial correlations in the SF positions, i.e. a randomly faulted $3 \mathrm{C}$ crystal is obviously unable to yield the expected $6 \mathrm{H}$ stacking. The concept of non-random faulting applied to the study of the $3 \mathrm{C}-6 \mathrm{H}$ has been introduced in Ref. 16 where different transformation mechanisms have been investigated: the layer displacement mechanism where the re-stacking occurs by diffusional rearrangement, and a dislocation-based mechanism where the re-stacking occurs through the glide of partial dislocations ${ }^{17}$. We make use of this formalism in combination with the previous equation in order to determine the transformation mechanism as well as the transformation level. The simulation results are shown in figure 3(c). It unambiguously appears that the $3 \mathrm{C}-6 \mathrm{H}$ transformation occurs through the glide of partial dislocations and the transformation level is $5.2 \%$. Prior to thermal annealing the transformation level was $1.6 \%$, which demonstrates that the $3 \mathrm{C}$ phase is indeed unstable at high temperatures $\left(\geq 1700^{\circ} \mathrm{C}\right)$. However, since this transformations requires partial dislocations to take place, a 
possible mean to grow large high-quality $3 \mathrm{C}-\mathrm{SiC}$ single crystals is hence to limit the formation of dislocations in the crystal, in particular by avoiding mechanical stresses during the growth.
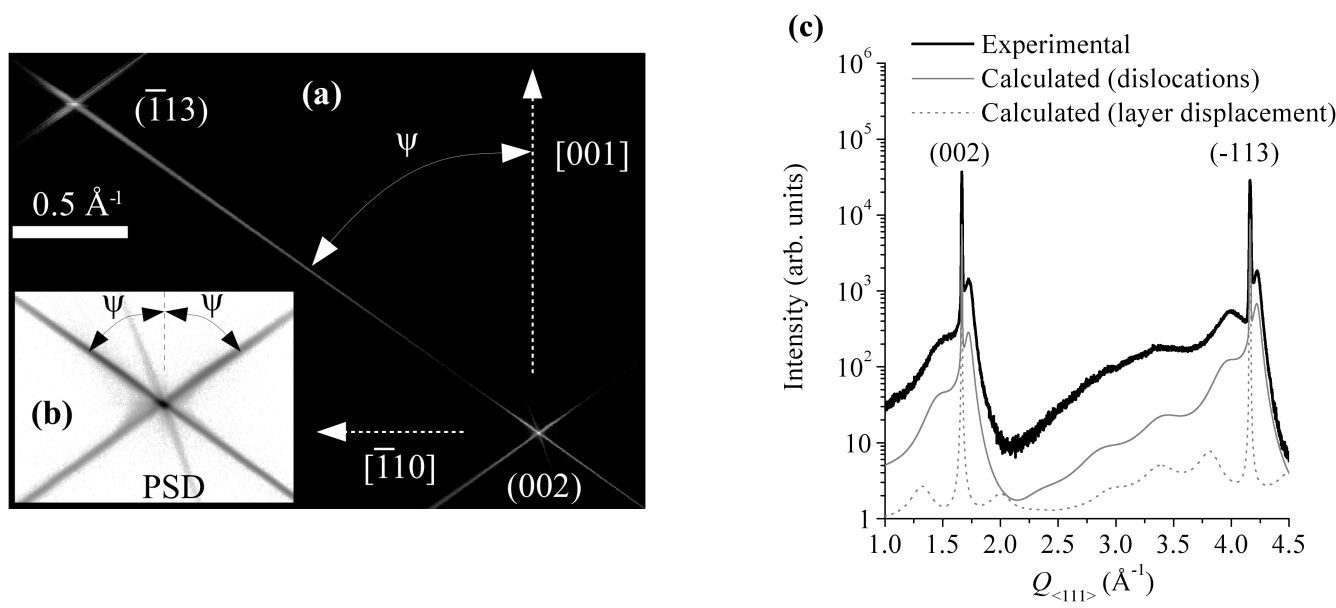

FIGURE 3. (a) wide-range RSM of an transformed $3 \mathrm{C}-\mathrm{SiC}$ crystal. (b) $12 \mathrm{x}$ magnification of the (002) reflection. (c) Simulation of the intensity distribution along the the diffuse streak. The curves are shifted vertically for clarity.

\section{CONCLUSIONS}

Stacking faults in 3C-SiC single crystals have been investigated by means of diffuse X-ray scattering. Widerange reciprocal space maps have been recorded on a laboratory-based diffractometer. A qualitative interpretation of the RSMs allows to detect the presence of SFs and spatial correlations (i.e. partially transformed crystals). The simulation of the diffuse scattering intensity distribution allows to determine the SF density, the transformation mechanism and the transformation level.

\section{ACKNOWLEDGEMENTS}

D.D. is grateful to the Region Limousin (France) for its financial support. D.C. and I.G-S. thank the MINTEX French ANR program (contract number ANR-09-BLAN-0189-01) and the MANSiC - Marie Curie Research and Training Network (contract number MRTN-CT-2006-035735) for their financial supports.

\section{REFERENCES}

1. N. W. Jepps, T. F. Page, Proc. Cryst. Growth Charact. 7, 259-307 (1983).

2. W. F. Knippenberg, Philips Res. Rep. 18, 161-274 (1963).

3. V. Heine, C. Cheng, R. J. Needs, Mater. Sci. Eng. B 11, 55-60 (1992).

4. H. Jagodzinski, Kristallogr. 16, 1235-1246 (1971).

5. P. Krishna, R. C. Marshall, J. Cryst. Growth 11, 147-150 (1971).

6. M. Dudley, X. R. Huang, W. M. Vetter, J. Phys. D.: Appl. Phys. 36, A30-A36 (2003).

7. E. Polychroniadis, M. Syvajarvi, R. Yakimova, J. Stoemenos, J. Cryst. Growth 263, 68-75 (2004).

8. R. Püsche, M. Hundhausen, L. Ley, K. Semmelroth, F. Schmid, G. Pensl, H. Nagasawa, J. Appl. Phys. 96, $5569-5575$ (2004).

9. W. S. Yoo, H. Matsunami, J. Appl. Phys. 70, 7124-7131 (1991).

10. J. M Cowley, Diffraction Physics, Amsterdam: North-Holland (1975).

11. H. Nagasawa, K. Yagi, T. Kawahara, J. Cryst. Growth 237-239, 1244-1249 (2002).

12. A. Boulle, O. Masson, R. Guinebretière, A. Lecomte, A. Dauger, J. Appl. Crystallogr. 35, 606-614 (2002).

13 I. K. Robinson, Phys. Rev. B 33, 3830-3836(1986).

14. A. Boulle, D. Chaussende, L. Latu-Romain, F. Conchon, O. Masson, R. Guinebretière, Appl. Phys. Lett. 89, 091902 (2006).

15. A. Boulle, F. Conchon, R. Guinebretière, Acta Crystallogr. A 62, 11-20 (2006).

16. V. K. Kabra, D. Pandey, S. Lele, J. Mater. Sci. 21, 1654-1666 (1986).

17. A. Boulle, J. Aube, I. Galben-Sandulache, D. Chaussende, Appl. Phys. Lett. 94, 201904 (2009). 